\title{
Evaluation of Some Conducting Polymers as Novel Antioxidants for Rubber Vulcanizates
}

\author{
M. A. Abd El-Ghaffar, ${ }^{1}$ K. A. Shaffei, ${ }^{2}$ and Nourelhoda Abdelwahab ${ }^{1}$ \\ ${ }^{1}$ Department of Polymers and Pigments, National Research Centre, Cairo, Egypt \\ ${ }^{2}$ Chemistry Department, Faculty of Science Helwan University, Cairo, Egypt \\ Correspondence should be addressed to M. A. Abd El-Ghaffar; mghaffar50@yahoo.com
}

Received 2 July 2013; Revised 25 August 2013; Accepted 21 September 2013; Published 9 February 2014

Academic Editor: Ali Akbar Entezami

Copyright (C) 2014 M. A. Abd El-Ghaffar et al. This is an open access article distributed under the Creative Commons Attribution License, which permits unrestricted use, distribution, and reproduction in any medium, provided the original work is properly cited.

\begin{abstract}
Natural rubber (NR) and styrene-butadiene rubber (SBR) formulations containing polyaromatic and polyheterocyclic amine homopolymers and copolymers were prepared. The studied homopolymers named polythiophene (PT), poly(o-phenylene diamine) (Po-PDA) and copolymers named poly(aniline-co-m-toluidine) (PAn-co-mT), poly(aniline-co-o-phenylene diamine) (PAn-co-o-PDA), poly(aniline-co-thiophene) (PAn-co-T), poly(aniline-co-2-amino pyridine) (PAn-co-2APy), and poly(2-amino pyridine-co-o-phenylene diamine) (P2APy-co-o-PDA) have been prepared and characterized. The rheological characteristics and physicomechanical properties of the compounded rubber mixes and vulcanizates were investigated and determined. The effects of the prepared polymers on the ageing characteristics of corresponding vulcanizates were evaluated. It was found that the prepared polymers have shown better antioxidant efficiency than the conventional antioxidants phenyl $\beta$ naphthyl amine $(\mathrm{P} \beta \mathrm{N})$ and the polymerized-2,2,4-trimethyl-1,2-dihydroquinoline (TMQ) industrially used in addition to their safety and ecofriendliness to the environment.
\end{abstract}

\section{Introduction}

Natural rubber like any other organic polymer is susceptible to oxidative degradation, which results in loss of physical and mechanical properties. Its oxidation arises from the formation of peroxy-free radicals that can propagate scission reactions of the rubber molecule $[1,2]$. Antioxidants, either natural or synthetic, are essential additives to retard this oxidation reaction.

Antioxidants can be classified according to the way by which they interfere with the oxidation of the polymer as follows.

(a) Chain breaking donors (CB-D) are those which are capable of competing with the substrate $(\mathrm{RH})$ for the alkyl peroxy radicals [1]. The hindered phenols and aromatic amines such as substituted p-phenylene diamine [3] are the most important of these antioxidants. Some biological antioxidants such as tocopherols (vitamin E) also act by CB-D mechanism:

$$
\mathrm{ROO}^{\circ}+\underset{\text { (antioxidant) }}{\mathrm{AH}} \stackrel{\mathrm{CB}-\mathrm{D}}{\longrightarrow} \mathrm{ROOH}+\underset{\text { (stable radical) }}{\mathrm{A}^{\bullet}}
$$

(b) Chain breaking acceptors (CB-A) are antioxidants which can compete with oxygen for the alkyl radicals. Quinones [4], nitro compounds, and stable oxyradicals are considered as CB-A antioxidants (1):

$$
\mathrm{R}+\mathrm{Q} \stackrel{\mathrm{CB}-\mathrm{A}}{\longrightarrow} \mathrm{RQ}
$$

(c) Preventive antioxidants decompose hydroperoxides by a process which does not give rise to free radicals or that can stabilize the hydroperoxide. The phosphite esters and a whole family of sulfur-containing compounds are among this set of compounds [1,5-7] (Scheme 1).

Abad et al. [3] used natural antioxidants for radiation vulcanization of natural rubber; among these were cystine, tyrosine, asparagines, phenyl alanine, and alanine.

Kuriakose et al. [7] used rice bran oil as a novel compounding ingredient in sulfur vulcanization of natural rubber.

It is known that serious drawbacks of all antioxidants existing today are volatility and extractability. The decrease in concentration of the antioxidants impairs the resistance 

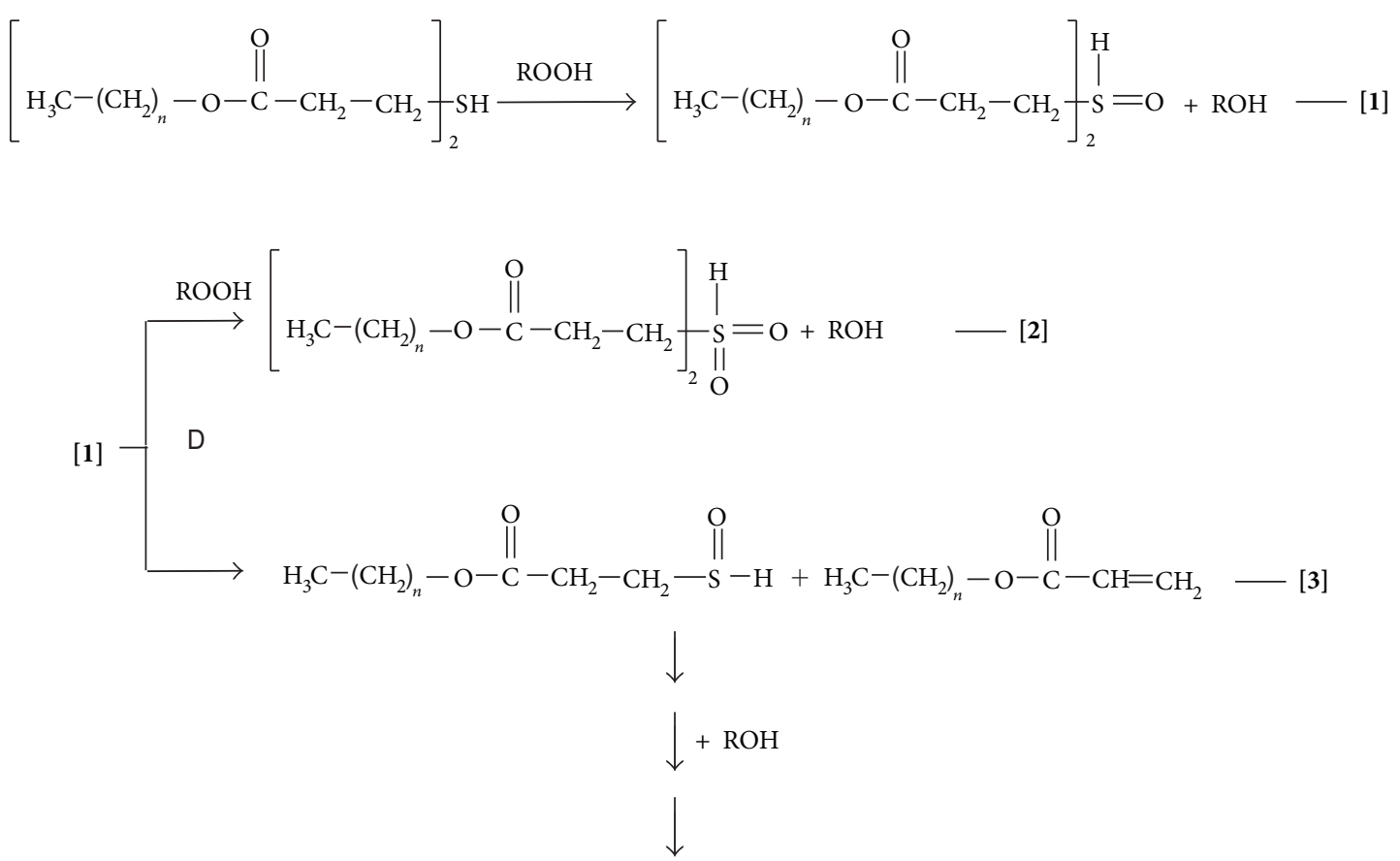

For example, $\mathrm{SO}_{2}, \mathrm{SO}_{3}, \mathrm{CH}_{2} \mathrm{SO}_{4}$

SCHEME 1

to degradation. One method to increase the persistence of the antioxidant in polymer is to use high molecular weight compounds which cannot migrate to the rubber surface and hence cannot be lost by volatilization [8].

Polyanilines are compounds which recently find wide applications. Polyanilines prepared by chemical oxidation processes for application as conducting polymers [9], and efficient corrosion inhibitors for steel protection [10], in addition to their synergistic antioxidant efficiency for NR and SBR vulcanizates as reported in our previous studies [11-13].

The present study aims to prepare and evaluate the previously mentioned homopolymers and copolymers of polyaromatic amines, polyheterocyclic compounds as antioxidants for SBR vulcanizates, and the role of chemical structure on their efficiencies.

\section{Experimental}

Two homopolymers, polythiophene (PT) and poly(ophenylene diamine) (Po-PDA), and five copolymers, poly (aniline-co-m-toluidine) (PAn-co-mT), poly (aniline-coo-phenylene diamine) (PAn-co-o-PDA), poly(aniline-cothiophene) (PAn-co-T), poly(aniline-co-2amino pyridine) (PAn-co-2APy), and poly (2-amino pyridine-co-o-phenylene diamine) (P2APy-Co-o-PD), were synthesized and characterized using spectrophotometric measurements (IR-UV-Vis) and thermal analysis. The methods of preparation and characterization were reported by Abd El-Ghaffar et al. in previous publications [8-13] and can be summarized as follows.

2.1. Preparation of (PT) and (Po-PDA) Homopolymers. $.01 \mathrm{~mol}$ of the conducting monomer in $50 \mathrm{~mL}$ of the suitable solvent and $0.01 \mathrm{~mol}$ of ammonium peroxydisulphate initiator was dissolved in $50 \mathrm{~mL}$ distilled water. The initiator solution was dropwised into the monomer solution with continuous stirring for two hours at temperature range $0-5^{\circ} \mathrm{C}$.

The precipitated conducting polymer was left for 24 hours, filtered, and washed several times with distilled water, methanol and dried at $50^{\circ} \mathrm{C}$ under vacuum. The yield $\geq 90 \%$ was characterized, examined for physical properties, and evaluated as antioxidants for NR and SBR vulcanizates.

2.2. Preparation of Various Conducting Copolymers. $0.05 \mathrm{~mol}$ of aniline in $50 \mathrm{~mL}$ methanol and $0.05 \mathrm{~mol}$ of m-toluidine in $50 \mathrm{~mL}$ methanol were mixed. To this blend of the two conducting monomer solutions was added dropwise with continuous stirring at $0-5^{\circ} \mathrm{C}(0.1 \mathrm{~mol}, 22.819 \mathrm{gm})$ of ammonium peroxydisulphate initiator in $50 \mathrm{~mL}$ distilled water with continuous stirring for 6 hours and left overnight. The copolymer was filtered off and the precipitate was washed several times with distilled water, methanol, and ethanol; after that the precipitate was subjected for drying under vacuum in an electric oven at $50^{\circ} \mathrm{C}$. The precipitate was characterized, examined for physical properties, and evaluated as antioxidant for NR and SBR vulcanizates.

The previous steps were repeated for all previously mentioned copolymers.

The rubber samples used in this investigation were natural rubber (SMR-20) and styrene-butadiene rubber (SBR1502).

The accelerator was $N$-cyclohexyl-2-benzothiazole sulphenamide (CBS), and the activator used in this study was stearic acid and zinc oxide.

Elemental sulfur was used as curing agent. 


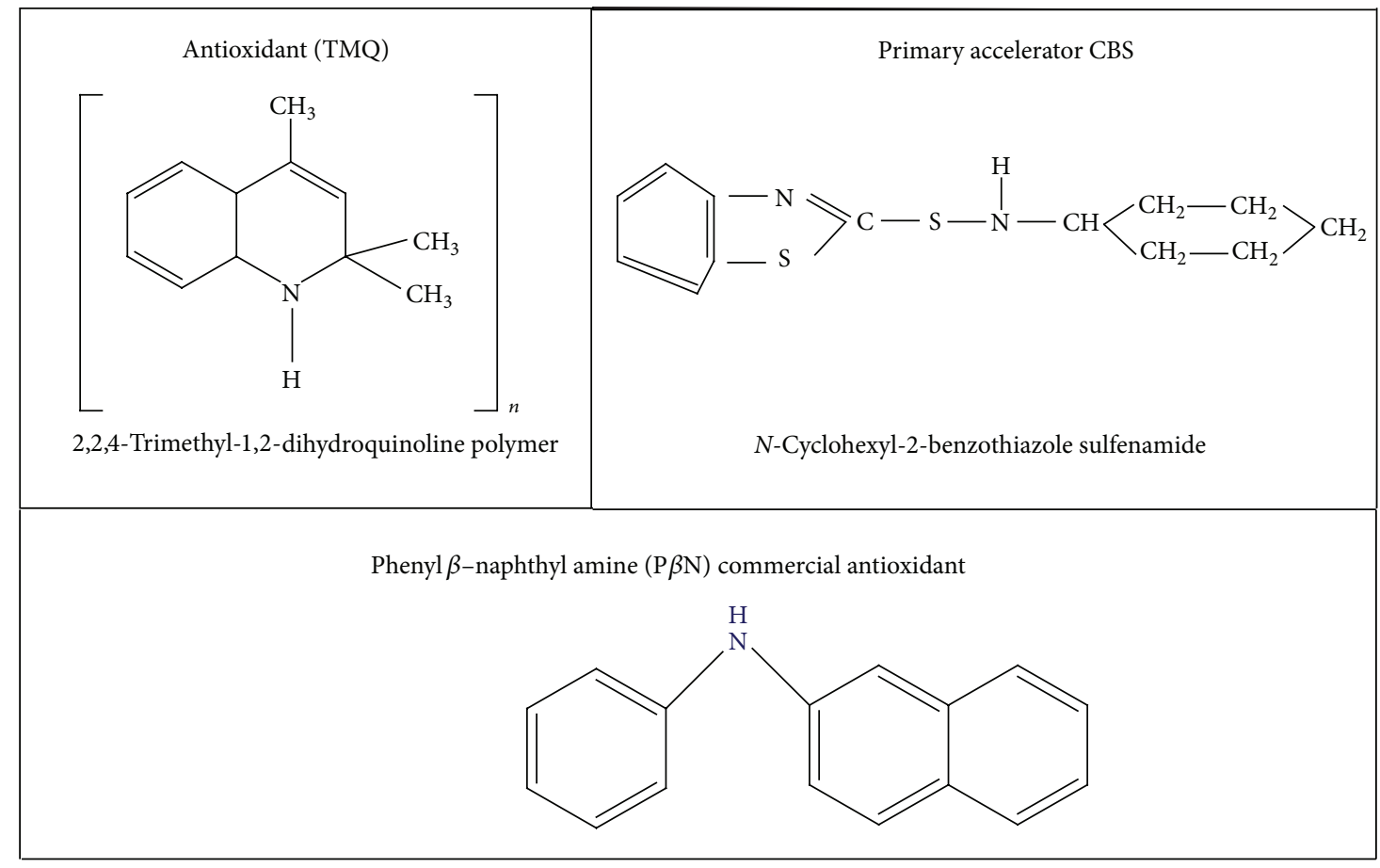

SCHEME 2

The mixes were prepared on a two-roll mill. The rheometric properties of the rubber mixes were determined by using Monsanto Oscillating Disc Rheometer [14]. The compounded rubber was vulcanized in a hydraulic press at $152^{\circ} \mathrm{C}$. The physicomechanical characteristics were determined according to standard methods [15]. The vulcanizates were subjected to thermal oxidative ageing [16] in an oven at $90^{\circ} \mathrm{C}$ for different time periods. The ageing data were compared with those of vulcanizates containing the antioxidant phenyl $\beta$ naphthyl amine $(\mathrm{P} \beta \mathrm{N})$. The equilibrium swelling was carried out in toluene [17] according to ASTM method D471 (2006).

The conducting polymers showing the best antioxidant results for NR and SBR vulcanizates were tested for ageing and subjected to UV for $72 \mathrm{hr}$ using UV lamp (120 w), in comparison with the antioxidants $(\mathrm{P} \beta \mathrm{N})$ and 2,2,4-trimethyl-1,2dihydroquinoline polymer (TMQ) as commercial stabilizers (Scheme 2).

\section{Results and Discussion}

3.1. Effect of the Prepared Homopolymers and Copolymers on the Properties of Natural Rubber Mixes. The prepared homopolymers and copolymers were incorporated in natural rubber mixes in a suitable dose (1 phr) compared with phenyl$\beta$-naphthyl amine $(\mathrm{P} \beta \mathrm{N})$, the commercial antioxidant which is widely used in rubber industry.

The mixing process was carried out on a laboratory mill according to the usual regime. The rubber formulations are given in Table 1.

The rheological characteristics of the mixes were determined using an oscillating disc rheometer at $142 \pm 1^{\circ} \mathrm{C}$ and are given in Table 2.
TABLE 1: Rubber formulations containing the prepared homopolymers and copolymers.

\begin{tabular}{lccccccccc}
\hline Formulation no. & 1 & 2 & 3 & 4 & 5 & 6 & 7 & 8 & 9 \\
\hline Control (without) & - & - & - & - & - & - & - & - & - \\
$\mathrm{P} \beta \mathrm{N}$ & - & 1 & - & - & - & - & - & - & - \\
$\mathrm{Po-PDA}$ & - & - & 1 & - & - & - & - & - & - \\
$\mathrm{PT}$ & - & - & - & 1 & - & - & - & - & - \\
$\mathrm{P}($ An-co-mT $)$ & - & - & - & - & 1 & - & - & - & - \\
$\mathrm{P}($ An-co-T $)$ & - & - & - & - & - & 1 & - & - & - \\
$\mathrm{P}($ An-co-o-PDA $)$ & - & - & - & - & - & & 1 & - & - \\
$\mathrm{P}($ An-co-2APy) & - & - & - & - & - & & - & 1 & - \\
$\mathrm{P}(2 \mathrm{APy}-\mathrm{Ao-o}-\mathrm{PDA})$ & - & - & - & - & - & & - & - & 1 \\
\hline
\end{tabular}

Base recipe (phr): NR, 100; stearic acid, 2; zinc oxide, 5; HAF, 30; processing oil, 3; CBS, 1; S, 2.

The physicomechanical properties of the vulcanizates were determined and are also given in Table 2.

From these data, it is clear that the prepared homopolymers and copolymers had an accelerating effect as shown from the decrease in the optimum cure time and the increase in cure rate index. On the other hand, it is obvious from the results of mechanical properties given also in Table 2 and illustrated in Figures 1, 2, and 3 that the prepared homopolymers and copolymers increased stress at yield, stress at rupture, strain at yield, and strain at rupture while decreasing equilibrium swelling which indicates that these polymers can act also as secondary stabilizers.

3.2. Effect of the Prepared Homopolymers and Copolymers as Antioxidants in Natural Rubber Mixes. Oxidative ageing 
TABLE 2: Rheometric characteristics and physico-mechanical properties of NR vulcanizates containing the prepared homopolymers and copolymers.

\begin{tabular}{|c|c|c|c|c|c|c|c|c|c|}
\hline \multirow{2}{*}{ Property } & \multicolumn{9}{|c|}{ Formulation no. } \\
\hline & 1 & 2 & 3 & 4 & 5 & 6 & 7 & 8 & 9 \\
\hline \multicolumn{10}{|c|}{ Rheometric characteristics at $142^{\circ} \mathrm{C} \pm 1^{\circ} \mathrm{C}$} \\
\hline Minimum torque, $M_{L}, \mathrm{dN} \cdot \mathrm{m}$ & 1.5 & 1 & 1 & 1 & 1.5 & 1 & 1 & 1 & 1.5 \\
\hline Maximum torque, $M_{H}, \mathrm{dN} \cdot \mathrm{m}$ & 64 & 66 & 66 & 70 & 68 & 72 & 68 & 67 & 66 \\
\hline Optimum cure time, $t_{c 90}, \min$ & 18 & 15 & 15 & 12 & 12 & 11 & 13 & 14 & 14 \\
\hline Scorch time, $t_{s 2}$, min & 8 & 6.5 & 5 & 5 & 6 & 5 & 6.5 & 4.5 & 6 \\
\hline Cure rate index, CRT, $\min ^{-1}$ & 10 & 11.76 & 10 & 14.29 & 16.67 & 16.67 & 15.38 & 11.76 & 12.5 \\
\hline \multicolumn{10}{|c|}{ Physicomechanical properties at the optimum cure time } \\
\hline Stress at yield, $\sigma_{B}, \mathrm{MPa}$ & 20.4 & 22.3 & 22 & 25.36 & 23.23 & 28.11 & 23.2 & 22.52 & 22.18 \\
\hline Stress at rupture, $\sigma_{R}, \mathrm{MPa}$ & 19.3 & 21.2 & 21.8 & 25.11 & 23.11 & 27.4 & 22.05 & 22.33 & 21.83 \\
\hline Strain at yield, $\varepsilon_{B}, \%$ & 496 & 530 & 525 & 645 & 539 & 650 & 535 & 530 & 530 \\
\hline Strain at rupture, $\varepsilon_{R}, \%$ & 503 & 540 & 530 & 647 & 541 & 670 & 540 & 533 & 531 \\
\hline Equilibrium swelling, Q\% & 325 & 270 & 259 & 225 & 232 & 219 & 243 & 248 & 252 \\
\hline
\end{tabular}

$M_{L}$ : minimum torque; $M_{H}$ : maximum torque; $t_{c 90}$ : cure time to $90 \%$ of full torque development.

$t_{c 90}$ : cure time; $t_{s 2}$ : scorch time to 2 units of torque increase above minimum torque.

CRI: cure rate index: $100 /\left(t_{c 90}-t_{s 2}\right)$.

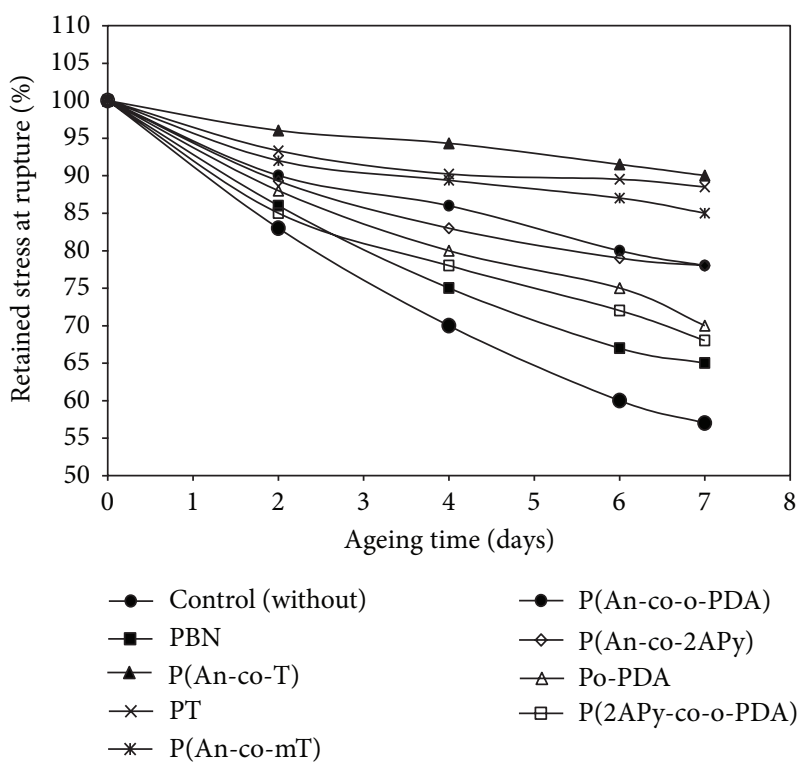

FIGURE 1: Retained strain at rupture versus ageing time for NR vulcanizates.

of elastomers is a very serious problem; during the ageing process, small amounts of oxygen are absorbed by natural or synthetic rubbers or by their vulcanizates. This causes a considerable change in their physicomechanical properties; consequently, it is necessary to protect rubber articles from oxidative ageing by adding compounds called "antioxidants" to rubber mixes.

The addition of antioxidants to rubber mixes can prolong the service life of the rubber vulcanizates; however, the efficiency of an antioxidant depends on different factors such as the type of rubber, the type of ageing, and the nature of the other rubber ingredients in the mix.

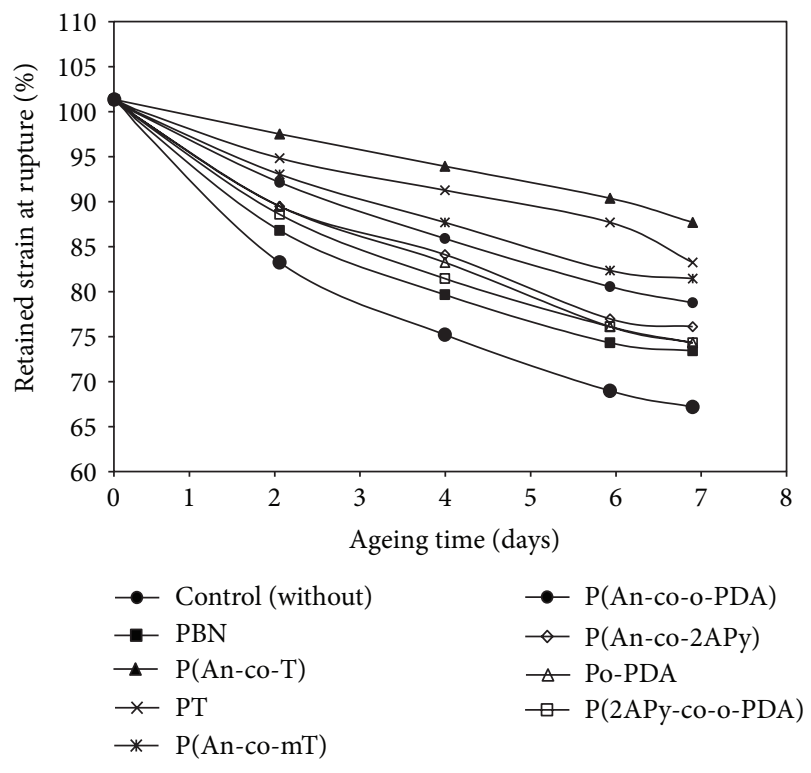

FIGURE 2: Retained stress at rupture versus ageing time for NR vulcanizates.

The efficiency of the antioxidants also greatly depends on their chemical structure.

In view of the above, the natural rubber mixes shown in Table 1 were vulcanized at $142 \pm 1^{\circ} \mathrm{C}$ for their optimum cure time. The rubber samples were subjected to thermal oxidative ageing in an oven at $90^{\circ} \mathrm{C} \pm 1^{\circ} \mathrm{C}$ for different time periods.

The physicomechanical properties of the aged samples were determined and the retained values of each property were calculated and represented in Figures 2 and 3. From these figures, it is clear that the retained values of tensile strength and elongation at break depend on the ageing time.

From these results, it is obvious that the prepared homopolymers and copolymers are more efficient to protect 


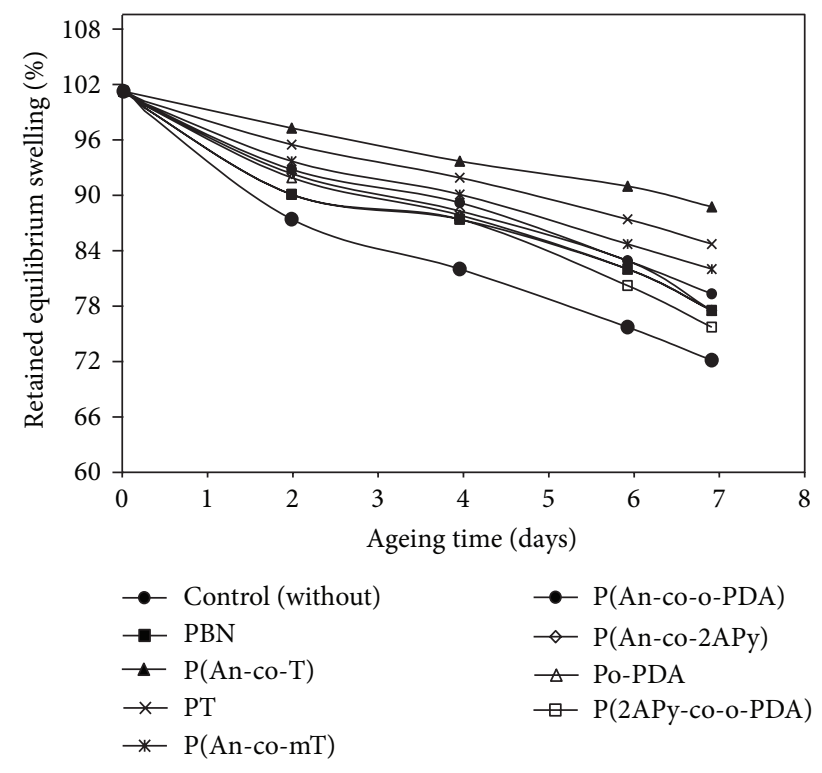

FIGURE 3: Retained equilibrium swelling versus ageing time for NR vulcanizates.

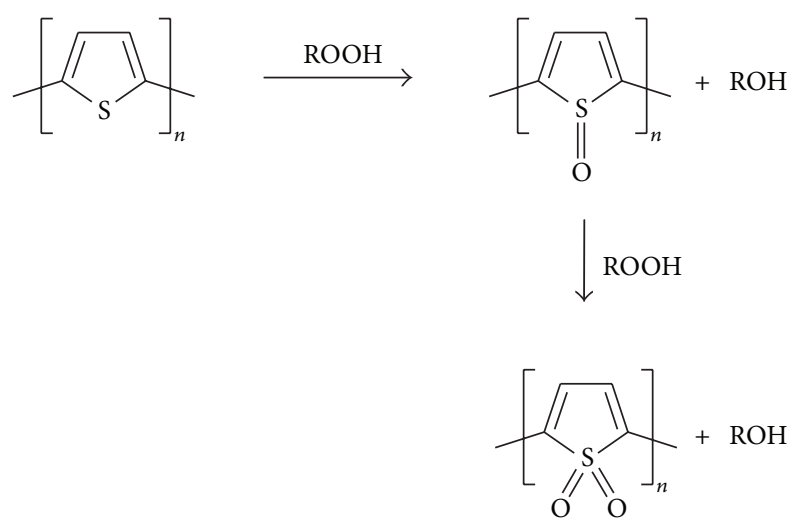

SCHEME 3: Mechanism of polythiophene as an antioxidant (hydroperoxide decomposition reactions in presence of polythiophene).

natural rubber vulcanizates against thermal ageing, practically to higher levels than the widely used antioxidant PBN and to some extent in case of TMQ.

Figure 3 depicts the dependence of the retained values of equilibrium swelling in toluene as a function of ageing time.

From the obtained results the efficiency of the prepared homopolymers and copolymers can be arranged in the following order:

$\mathrm{P}($ An-co-T $)>\mathrm{PT}>\mathrm{P}($ An-co-mT $)>\mathrm{P}($ An-co-o-PDA $)>$ $\mathrm{P}($ An-co-2APy $)>\mathrm{P}($ o-PDA-co-2APy $)>$ Po-PDA $=\mathrm{P} \beta \mathrm{N}>$ control (without antioxidant).

It is quite evident that poly(aniline-co-thiophene) had the highest efficiency as antioxidant and this is due to a mixed mechanism including the liability of hydrogen in polyaniline moiety which blocks the peroxide radicals in addition to hydroperoxide decomposition in case of the polythiophene moiety. These results are in accordance with the results reported by Drake et al. [18] (Scheme 3).
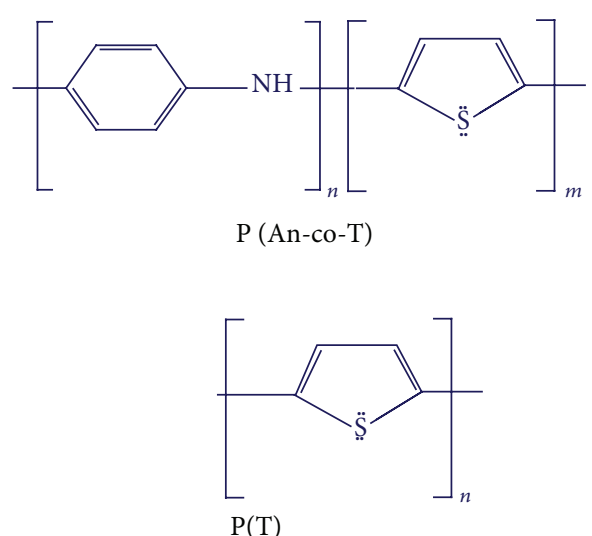

SCHEME 4

TABLE 3: SBR formulations containing the prepared homopolymers and copolymers.

\begin{tabular}{lccccccccc}
\hline Formulation no. & $1^{\prime}$ & $2^{\prime}$ & $3^{\prime}$ & $4^{\prime}$ & $5^{\prime}$ & $6^{\prime}$ & $7^{\prime}$ & $8^{\prime}$ & $9^{\prime}$ \\
\hline Control (without) & - & - & - & - & - & - & - & - & - \\
$\mathrm{P} \beta \mathrm{N}$ & - & 1 & - & - & - & - & - & - & - \\
$\mathrm{Po-PDA}$ & - & - & 1 & - & - & - & - & - & - \\
$\mathrm{PT}$ & - & - & - & 1 & - & - & - & - & - \\
$\mathrm{P}($ An-co-mT $)$ & - & - & - & - & 1 & - & - & - & - \\
$\mathrm{P}($ An-co-T $)$ & - & - & - & - & - & 1 & - & - & - \\
$\mathrm{P}($ An-co-o-PDA $)$ & - & - & - & - & - & - & 1 & - & - \\
$\mathrm{P}($ An-co-2APy) & - & - & - & - & - & - & - & 1 & - \\
$\mathrm{P}(2 \mathrm{APy}-\mathrm{AO}-\mathrm{O}-\mathrm{PDA})$ & - & - & - & - & - & - & - & - & 1 \\
\hline
\end{tabular}

Base recipe (phr): SBR, 100; stearic acid, 2; zinc oxide, 5; HAF, 30; processing oil, 3; CBS, 1 ; S, 2 .

On the other hand, polythiophene also produces good results due to high conjugation of thiophene ring which attracts the lone pair of electrons of sulfur to a great extent, which causes deficiency of electrons on sulfur atom, so that sulfur atom could capture the growing radicals formed on rubber molecules stopping the oxidation of rubber.

The structures of poly(aniline-co-thiophene) $\mathrm{P}(\mathrm{An}-\mathrm{co}-\mathrm{T})$ and polythiophene (PT) are illustrated in Scheme 4.

\subsection{Effect of the Prepared Homopolymers and Copolymers} on the Properties of Styrene-Butadiene Rubber (SBR). The prepared homopolymers and copolymers were incorporated in SBR mixes and compared with the commercial antioxidant $\mathrm{P} \beta \mathrm{N}$ as shown in Table 3 .

The rheometer characteristics of the rubber mixes were determined at $152^{\circ} \mathrm{C} \pm 1$ using Monsanto Oscillating Disc Rheometer 100 . The samples were vulcanized at $152 \pm 1^{\circ} \mathrm{C}$ for their optimum cure time and their physicomechanical properties were determined.

Table 4 shows the rheometer characteristics as well as the physicomechanical properties of the rubber samples.

From the data given in Table 4 , it is clearly seen that the prepared homopolymers and copolymers decreased the optimum cure time and increased the cure rate index. This indicates that these compounds had an accelerating effect. 
TABLE 4: Rheometric characteristics and physico-mechanical properties of SBR vulcanizates containing the prepared homopolymers and copolymers.

\begin{tabular}{|c|c|c|c|c|c|c|c|c|c|}
\hline \multirow{2}{*}{ Property } & \multicolumn{9}{|c|}{ Formulation no. } \\
\hline & $1^{\prime}$ & $2^{\prime}$ & $3^{\prime}$ & $4^{\prime}$ & $5^{\prime}$ & $6^{\prime}$ & $7^{\prime}$ & $8^{\prime}$ & $9^{\prime}$ \\
\hline \multicolumn{10}{|c|}{ Rheometric characteristics at $152 \pm 1^{\circ} \mathrm{C}$} \\
\hline Minimum torque, $M_{L}, \mathrm{dN} \cdot \mathrm{m}$ & 13 & 12 & 12 & 11 & 11 & 11 & 11 & 11 & 12 \\
\hline Maximum torque, $M_{H}, \mathrm{dN} \cdot \mathrm{m}$ & 65 & 69 & 65 & 74 & 72 & 79.5 & 71.5 & 69 & 69 \\
\hline Optimum cure time, $t_{c 90}$, min & 25 & 22.5 & 24 & 18 & 18 & 17 & 19 & 21 & 23 \\
\hline Scorch time, $t_{s 2}$, min & 5.5 & 5.0 & 6.0 & 4.0 & 4.0 & 3.0 & 4.5 & 5.5 & 5.5 \\
\hline Cure rate index, CRT, $\min ^{-1}$ & 5.13 & 5.71 & 5.56 & 7.14 & 7.14 & 7.14 & 6.9 & 6.45 & 5.71 \\
\hline \multicolumn{10}{|c|}{ Physicomechanical properties at the optimum cure time } \\
\hline Stress at yield, $\sigma_{B}, \mathrm{~Pa}$ & 15.28 & 17.2 & 17.58 & 24.4 & 22.35 & 26.12 & 21.8 & 19.5 & 18.86 \\
\hline Stress at rupture, $\sigma_{R}, \mathrm{MPa}$ & 15.01 & 16.34 & 17.06 & 23.83 & 21.4 & 25.8 & 20.2 & 18.47 & 17.2 \\
\hline Strain at yield, $\varepsilon_{B}, \%$ & 325 & 368 & 370 & 451 & 430 & 480 & 423 & 410 & 380 \\
\hline Strain at rupture, $\varepsilon_{R}, \%$ & 338 & 372 & 375 & 460 & 444 & 483 & 420 & 417 & 383 \\
\hline Equilibrium swelling, Q\% & 335 & 318 & 283 & 240 & 251 & 234 & 259 & 268 & 275 \\
\hline
\end{tabular}

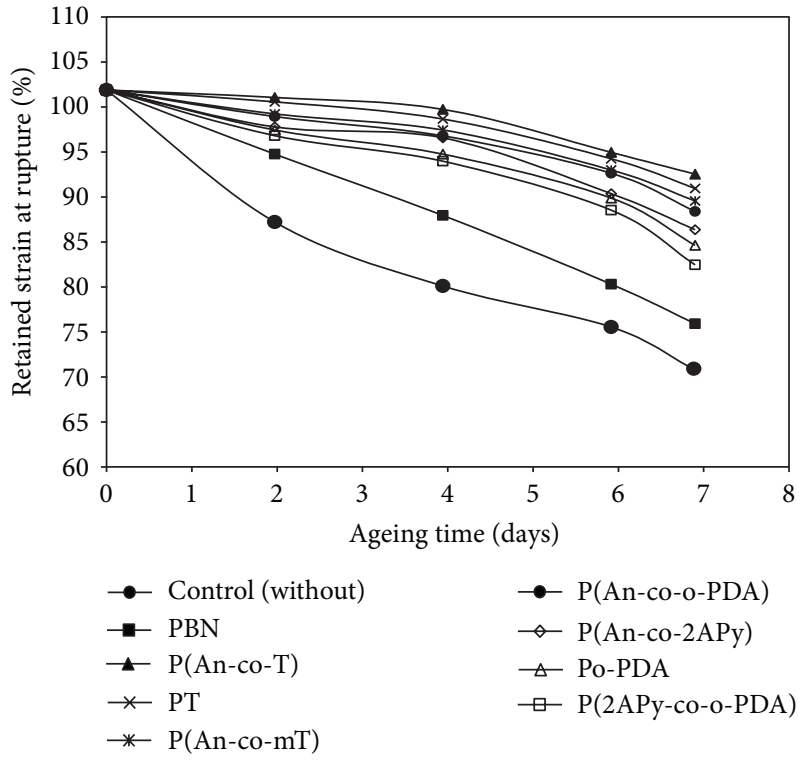

FIGURE 4: Retained strain at rupture versus ageing time for SBR vulcanizates.

Also, it is clearly noticed that the prepared polymers increased the stress at yield, stress at rupture, strain at yield, and strain at rupture while they decreased the equilibrium swelling.

From these results, the prepared polymers may be used as antioxidants in SBR vulcanizates. This is due to the presence of reactive centers in their chemical structures, in addition to double bonds.

Thus, the evaluation of the prepared homopolymers and copolymers as antioxidants for SBR vulcanizates is very interesting.

3.4. Effect of the Prepared Homopolymers and Copolymers as Antioxidants in Styrene-Butadiene Rubber (SBR) Mixes. From the previous study, it was found that the prepared

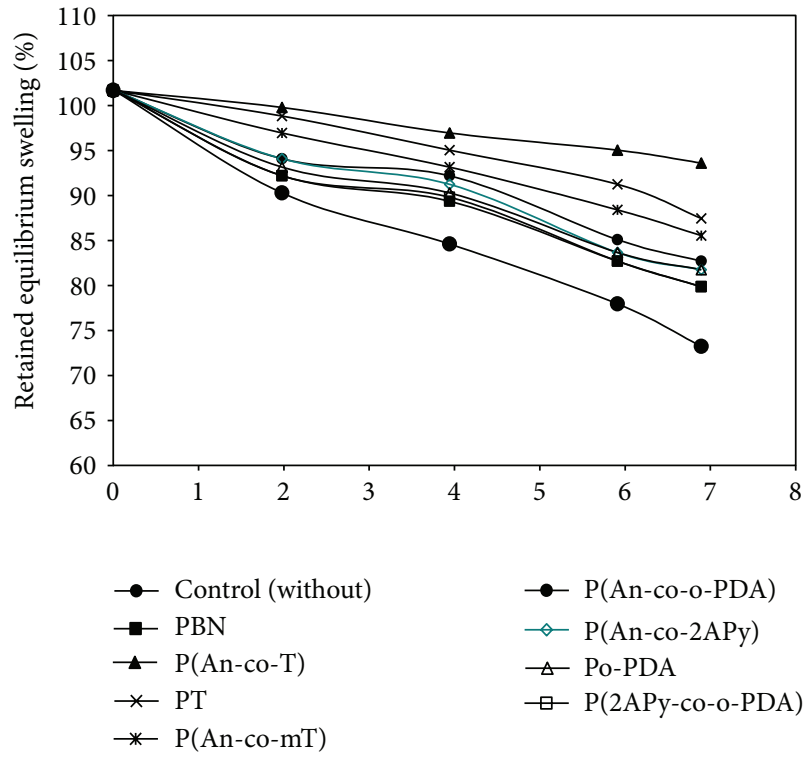

FIGURE 5: Retained stress at rupture versus ageing time for SBR vulcanizates.

homopolymers and copolymers proved themselves to be compounds of the highest efficiency as antioxidants in SBR vulcanizates. Thus, it is of great interest to study the effect of these compounds as antioxidants for the synthetic styrenebutadiene rubber.

The rubber samples were vulcanized at their optimum cure time. The vulcanizates were subjected to thermal ageing at $90^{\circ} \mathrm{C}$ in an electric oven, as previously described, for different periods of time.

The physicomechanical properties of the aged samples were determined and the retained values of each property were calculated.

Figures 4 and 5 represent the dependence of both stress and strain at rupture on the ageing time for rubber samples containing the investigated homopolymers and copolymers. 


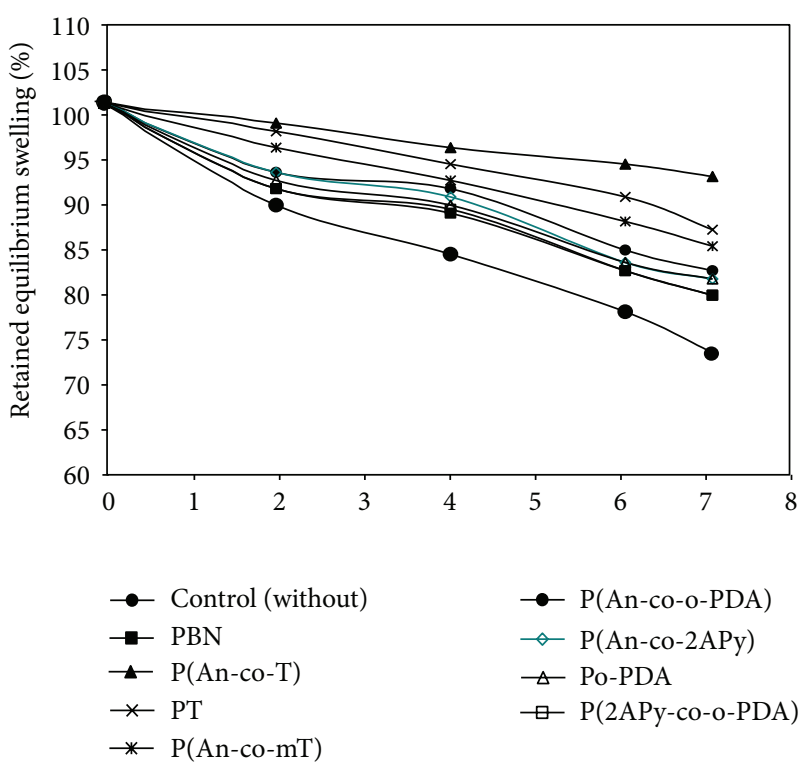

FIGURE 6: Retained equilibrium swelling versus ageing time for SBR vulcanizates.

TABLE 5: NR formulations containing the prepared $\mathrm{P}(\mathrm{An}-\mathrm{co}-\mathrm{T})$ and PT.

\begin{tabular}{lccccc}
\hline Formulation no. & 10 & 11 & 12 & 13 & 14 \\
\hline Control (without) & - & - & - & - & - \\
P $\beta \mathrm{N}$ & - & 1 & - & - & - \\
TMQ & - & - & 1 & - & - \\
P(An-co-T) & - & - & - & 1 & - \\
PT & - & - & - & - & 1 \\
\hline
\end{tabular}

Rheometric characteristics at $142 \pm 1^{\circ} \mathrm{C}$

$\begin{array}{lccccc}\text { Minimum torque, } M_{L}, \mathrm{dN} \cdot \mathrm{m} & 2 & 1 & 1 & 1 & 1 \\ \text { Maximum torque, } M_{H}, \mathrm{dN} \cdot \mathrm{m} & 70 & 69 & 69.5 & 70 & 72 \\ \text { Optimum cure time, } t_{c 90}, \mathrm{~min} & 13 & 12 & 11 & 12 & 11\end{array}$

Physicomechanical properties at the optimum cure time

Stress at rupture, $\sigma_{R}, \mathrm{MPa} \quad 17.84 \quad 18.75 \quad 19.8 \quad 27.4 \quad 25.10$

Strain at rupture, $\varepsilon_{R}, \% \quad 765 \quad 857 \quad 705 \quad 670 \quad 647$

Base recipe (phr): NR, 100; stearic acid, 2; zinc oxide, 5; HAF, 30; processing oil, 3; CBS, 1; S, 2.

The obtained results of equilibrium swelling as shown in Figure 6 also ensure the above results.

From the previous ageing results of NR and SBR, it is clearly seen that poly(aniline-co-thiophene) and polythiophene can be considered as the most efficient antioxidants in comparison with other prepared homopolymers and copolymers. These two polymers were examined for their antioxidant performance and UV stabilization in comparison with the commercial antioxidants $\mathrm{P} \beta \mathrm{N}$ and TMQ. The formulations are given in Tables 5 and 6 .

The results of retained stress at rupture and retained strain at rupture for aged NR and SBR vulcanizates are illustrated in Figures 7, 8, 9, and 10, respectively.

From these results it is clearly seen that the prepared polymers of $\mathrm{P}(\mathrm{An}-\mathrm{co}-\mathrm{T})$ and $\mathrm{PT}$ have shown outstanding properties which are more superior than the commercial
TABLE 6: SBR Formulations containing prepared $\mathrm{P}(\mathrm{An}-\mathrm{co}-\mathrm{T})$ and PT

\begin{tabular}{lccccc}
\hline Formulation no. & 15 & 16 & 17 & 18 & 19 \\
\hline Control (without) & - & - & - & - & - \\
$\mathrm{P} \beta \mathrm{N}$ & - & 1 & - & - & - \\
$\mathrm{TMQ}$ & - & - & 1 & - & - \\
$\mathrm{P}($ An-co-T) & - & - & - & 1 & - \\
$\mathrm{PT}$ & - & - & - & - & 1 \\
\hline \multicolumn{5}{c}{ Rheometric characteristics at $152 \pm 1^{\circ} \mathrm{C}$} \\
Minimum torque, $M_{L}, \mathrm{dN} \cdot \mathrm{m}$ & 2 & 1 & 1 & 1 & 1 \\
Maximum torque, $M_{H}, \mathrm{dN} \cdot \mathrm{m}$ & 70 & 69 & 69.5 & 70 & 72 \\
Optimum cure time, $t_{c 90}, \mathrm{~min}$ & 13 & 12 & 11 & 12 & 11 \\
\hline
\end{tabular}

Physicomechanical properties at the optimum cure time

$\begin{array}{llllll}\text { Stress at rupture, } \sigma_{R}, \mathrm{MPa} & 15.61 & 15.93 & 15.97 & 27.4 & 25.10\end{array}$

Strain at rupture, $\varepsilon_{R}, \% \quad 624 \quad 662 \quad 651 \quad 670 \quad 647$

Base recipe (phr): SBR, 100; stearic acid, 2; zinc oxide, 5; HAF, 30; processing oil, 3; CBS, 1; S, 2.

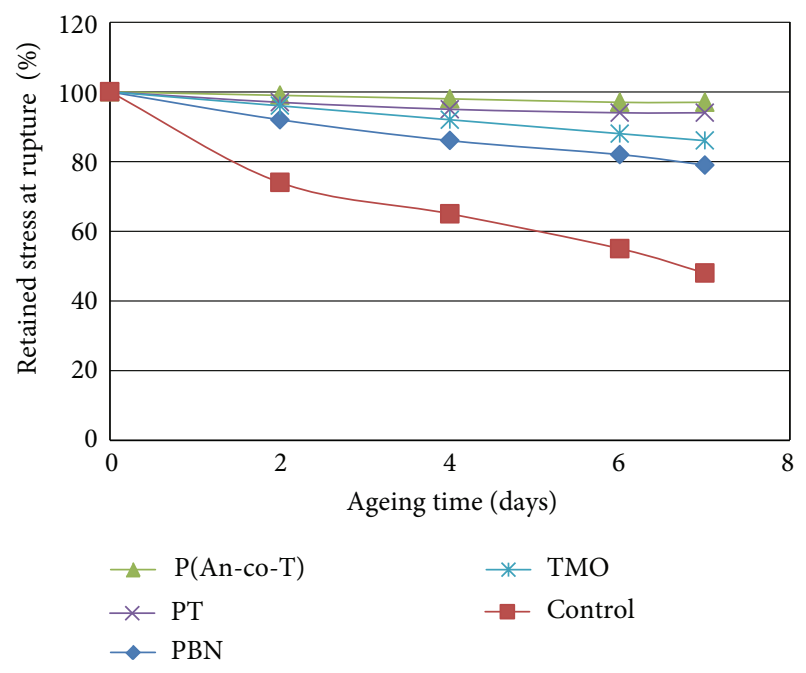

FIGURE 7: Retained stress at rupture versus ageing time for NR vulcanizates.

antioxidants $\mathrm{P} \beta \mathrm{N}$ and TMQ. These polymers are safe and environmentally acceptable $[8,10]$ and characterized with their easy method for preparation.

3.5. Effect of UV Exposure [19] on the Properties of Rubber Vulcanizates Containing $P(A n-c o-T)$ and $P T$ in Comparison with $P \beta N$ and TMQ. The rubber vulcanizates of control and those containing $1 \mathrm{phr}$ of the polymers $\mathrm{P}(\mathrm{An}-\mathrm{co}-\mathrm{T})$, PT compared with $\mathrm{P} \beta \mathrm{N}$ and TMQ were subjected to UV irradiation for $72 \mathrm{hrs}$. The mechanical properties of the exposed samples (mainly stress at rupture and strain at rupture) were examined and evaluated and showed better results than $\mathrm{P} \beta \mathrm{N}$ and TMQ. The data are given in Table 7.

\section{Conclusions}

Some conducting polymers include poly(aniline-co-thiophene), poly(aniline-co-2-amino pyridine), poly(2-amino 
TABLE 7: Retained stress and strain at rupture for NR and SBR vulcanizates containing the commercial antioxidants $\mathrm{P} \beta \mathrm{N}$, TMQ and prepared polymers of $\mathrm{P}(\mathrm{An}-\mathrm{co}-\mathrm{T})$ and $\mathrm{PT}$ after exposure to UV irradiation for $72 \mathrm{hr}$.

\begin{tabular}{|c|c|c|c|c|c|c|c|c|c|c|}
\hline \multirow{3}{*}{ Formulation property } & \multicolumn{5}{|c|}{ NR vulcanizates } & \multicolumn{5}{|c|}{ SBR vulcanizates } \\
\hline & Control & $\mathrm{P} \beta \mathrm{N}$ & TMQ & $\mathrm{P}(\mathrm{An}-\mathrm{co}-\mathrm{T})$ & PT & Control & $\mathrm{P} \beta \mathrm{N}$ & TMQ & $P(A n-c o-T)$ & PT \\
\hline & 10 & 11 & 12 & 13 & 14 & 15 & 16 & 17 & 18 & 19 \\
\hline Ret. stress at rupt., $\%$ after $72 \mathrm{hr}$ UV exposure & 89 & 98 & 100 & 100 & 100 & 90 & 99 & 93 & 100 & 100 \\
\hline Ret. strain at rupt., \% after $72 \mathrm{hr}$ UV exposure & 90 & 98 & 100 & 100 & 100 & 91 & 99 & 94 & 100 & 100 \\
\hline
\end{tabular}

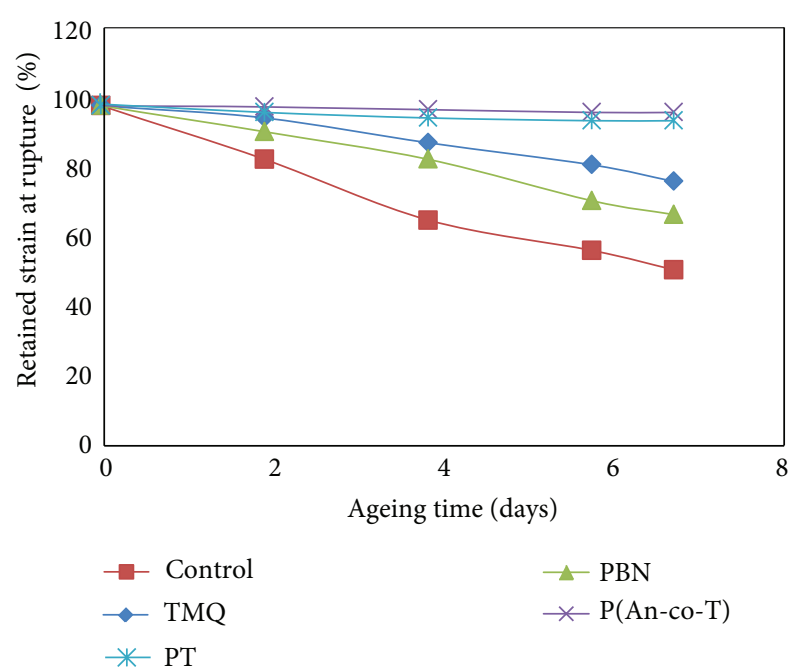

FIGURE 8: Retained strain at rupture versus ageing time for NR vulcanizates.

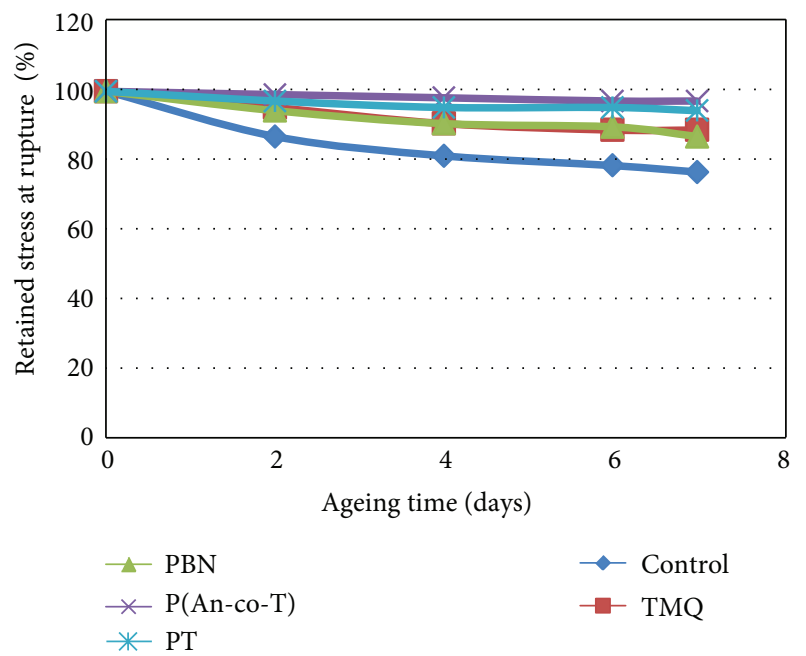

FIGURE 9: Retained stress at rupture versus ageing time for SBR vulcanizates.

pyridine-co-o-phenylene diamine), and poly(thiophene) were prepared and characterized. The polymers were examined for their antioxidant efficiency and UV protection for $\mathrm{NR}$ and SBR vulcanizates compared with the commercial antioxidants $\mathrm{P} \beta \mathrm{N}$ and TMQ. The prepared polymers of $\mathrm{P}($ An-co-T $)$ and $\mathrm{PT}$ showed high antioxidant performance exceeding the commercial antioxidants, in addition to

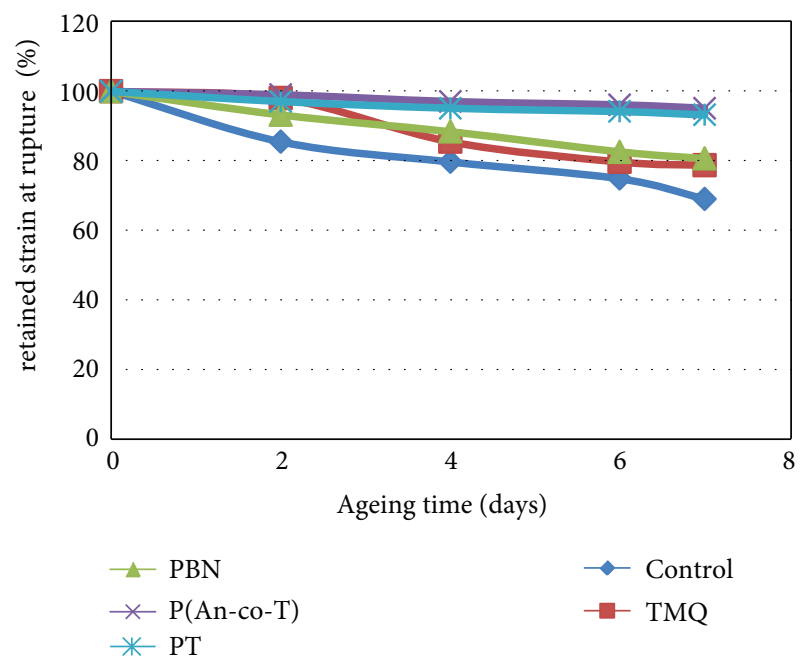

FIGURE 10: Retained strain at rupture versus ageing time for SBR vulcanizates.

their safety, environmental acceptance, and easy way of preparation. The data indicated also that the polymers can act as secondary accelerators in addition to their antioxidant efficiency.

\section{Conflict of Interests}

The authors declare that there is no conflict of interests regarding the publication of this paper.

\section{References}

[1] S. Al-Malaika, "Mechanisms of antioxidant action and stabilisation technology—-the Aston experience," Polymer Degradation and Stability, vol. 34, no. 1-3, pp. 1-36, 1991.

[2] D. Barnard and P. M. Lewis, "Oxidative aging," Natural Rubber Science \& Technology, pp. 621-675, 1988.

[3] L. V. Abad, L. S. Relleve, C. T. Aranilla, A. K. Aliganga, C. M. San Diego, and A. M. Dela Rosa, "Natural antioxidants for radiation vulcanization of natural rubber latex," Polymer Degradation and Stability, vol. 76, no. 2, pp. 275-279, 2002.

[4] M. W. Sabaa, T. M. Madkour, and A. A. Yassin, "Polymerization products of p-benzoquinone as bound antioxidants for blends of natural rubber," Polymer Degradation and Stability, vol. 26, no. 4, pp. 347-359, 1989.

[5] M. Giurginca, J. M. Herdan, L. Cira, G. Vǎleanu, and G. Ivan, "Grafted mercapto-1,3,5-triazinic antioxidants for elastomers," Polymer Degradation and Stability, vol. 36, no. 1, pp. 53-57, 1992. 
[6] H. Meier, P. Dubs, H. Künzi et al., "Some aspects of a new class of sulfur containing phenolic antioxidants," Polymer Degradation and Stability, vol. 49, no. 1, pp. 1-9, 1995.

[7] A. P. Kuriakose and G. Rajendran, "Rice bran oil as a novel compounding ingredient in sulphur vulcanization of natural rubber," European Polymer Journal, vol. 31, no. 6, pp. 595-602, 1995.

[8] M. N. Ismail, M. S. Ibrahim, and M. A. Abd El-Ghaffar, "Polyaniline as an antioxidant and antirad in SBR vulcanizates," Polymer Degradation and Stability, vol. 62, no. 2, pp. 337-341, 1998.

[9] M. A. Abd El-Ghaffar, N. A. Mohamed, A. A. Ghoneim, and K. A. Shaffei, "Characterization and electrical properties of some hydrochloric acid-doped aniline and 2-amino-pyridine homopolymers and copolymers," Polymer-Plastics Technology and Engineering, vol. 45, no. 12, pp. 1327-1338, 2006.

[10] M. A. Abd El-Ghaffar, E. A. M. Youssef, W. M. Darwish, and F. M. Helaly, "A novel series of corrosion inhibitive polymers for steel protection," Journal of Elastomers and Plastics, vol. 30, no. 1, pp. 68-94, 1998.

[11] F. M. Helaly, W. M. Darwich, and M. A. Abd El-Ghaffar, "Effect of some polyaromatic amines on the properties of NR and SBR vulcanizates," Polymer Degradation and Stability, vol. 64, no. 2, pp. 251-257, 1999.

[12] A. A. Wazzan, M. N. Ismail, and M. A. Abd El Ghaffar, "Evaluation of some polyaromatic amines as antirads and antifatigue agents in SBR vulcanizates," International Journal of Polymer Analysis and Characterization, vol. 10, no. 1-2, pp. 57-69, 2005.

[13] M. N. Ismail, M. A. Abd El Ghaffar, K. A. Shaffei, and N. A. Mohamed, "Some novel polyamines as antioxidants for SBR vulcanizates," Polymer Degradation and Stability, vol. 63, no. 3, pp. 377-383, 1999.

[14] ASTM D 2084-76T, "For determination of the rheometer characteristics using a Monsanto Oscillating Disc Rheometer, $100, " 1972$.

[15] ASTM D 412-66T, "For determination of Physico-mechanical properties using Zwick tensile testing machine. (model 1425)," 1967.

[16] ASTM D 573, "For carrying out the ageing of samples in an electric oven," 1952.

[17] ASTM D471, "For determination of swelling in toluene," 2006.

[18] W. O. Drake, J. R. Pauquet, J. Zingg, and H. Zweifel, "Stabilization of polymeric materials state-of-the-art, scope and limitations," Polymer Preprints, vol. 34, no. 2, p. 174, 1993.

[19] H. Zweifel, Stabilization of Polymeric Materials, Springer, Heidelberg, Germany, 1997. 

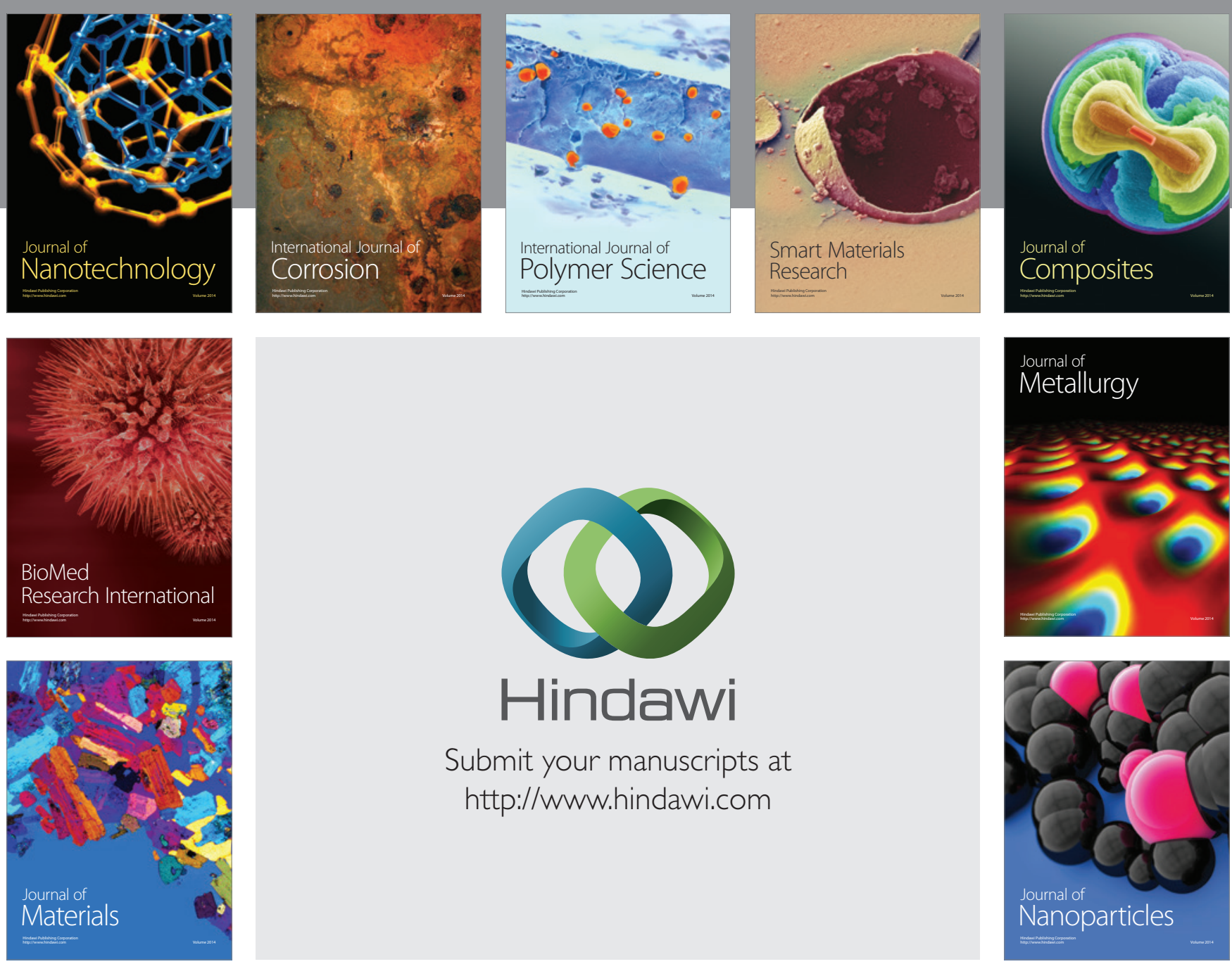

Submit your manuscripts at http://www.hindawi.com
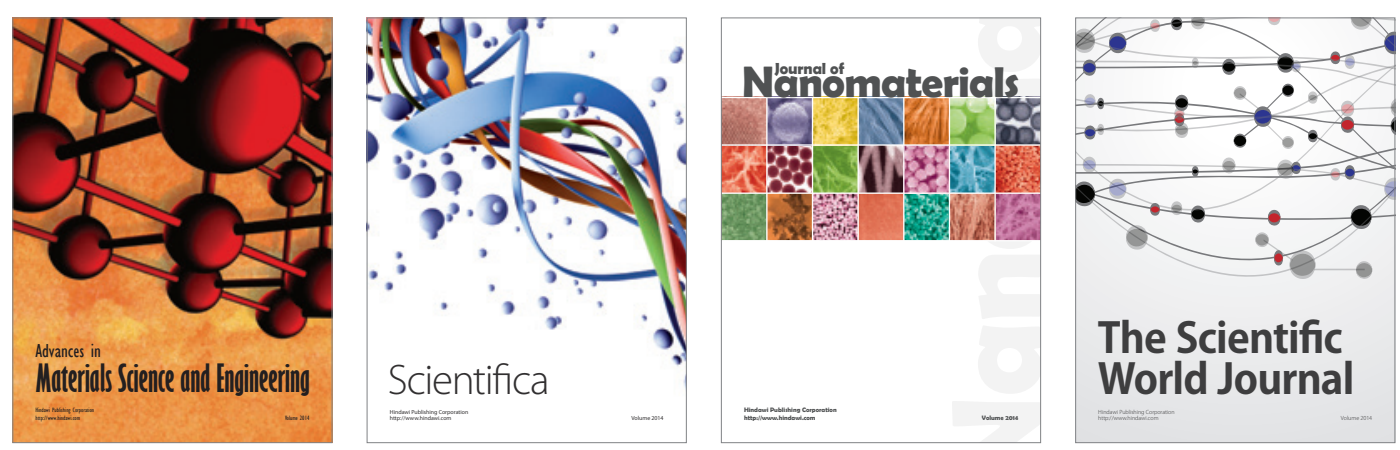

\section{The Scientific World Journal}
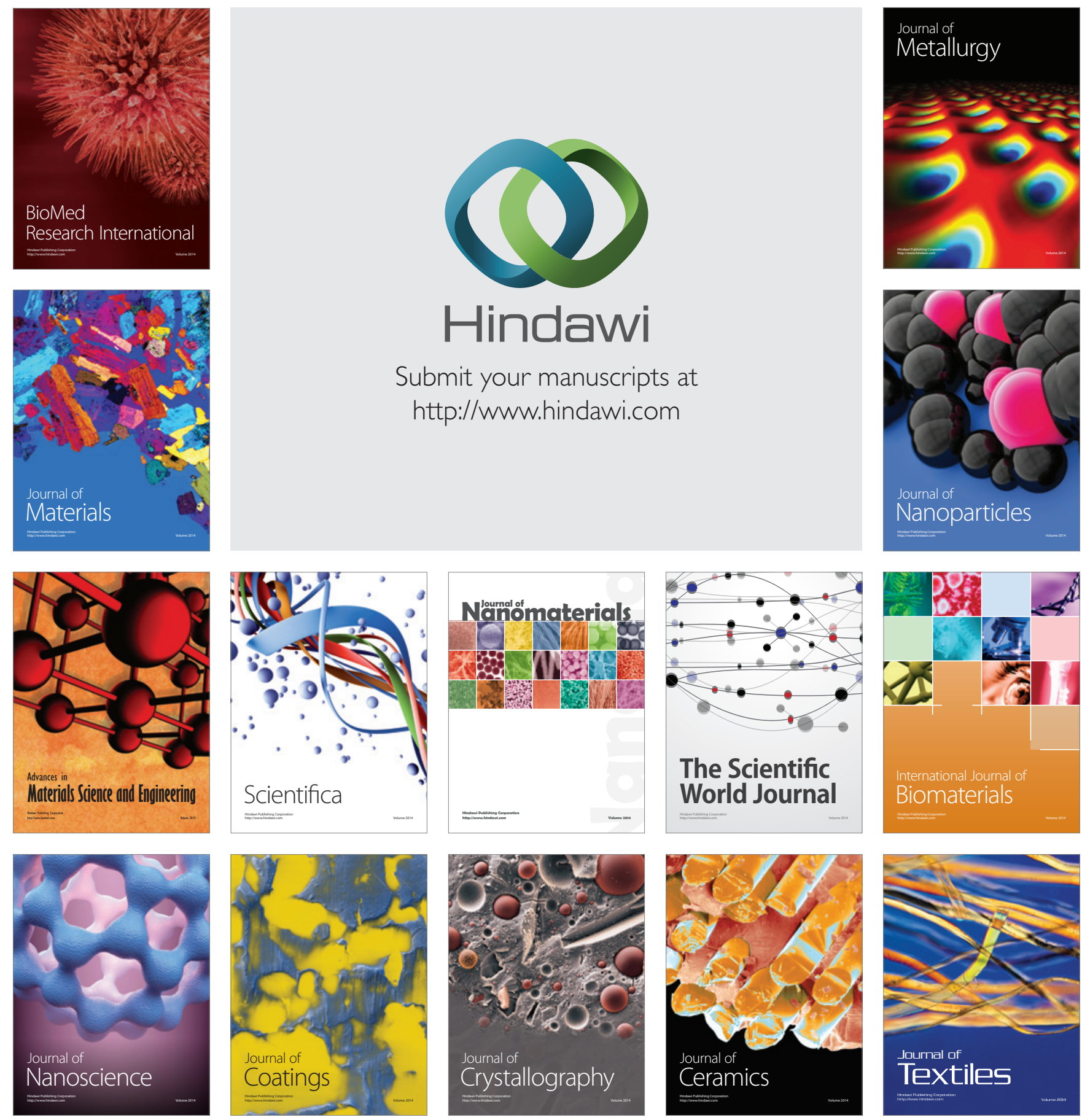